\title{
Diagnostic Problems of a Male Patient with Mixed Connective Tissue Disease
}

\author{
Nina Oktafianti Marfu'ah1', Awalia ${ }^{2 *}$ \\ ${ }^{1}$ Department of Internal Medicine, Faculty of Medicine, Universitas Airlangga - Dr. Soetomo General Hospital Surabaya, \\ Indonesia.
}

${ }^{2}$ Division of Rheumatology, Department of Internal Medicine, Faculty of Medicine, Universitas Airlangga - Dr. Soetomo

General Hospital Surabaya, Indonesia.

\begin{abstract}
A B S T R A C T
Mixed connective tissue disease (MCTD) is an overlap disease, recognized as an entity disease with a mixture of clinical manifestations from systemic lupus erythematosus (SLE), systemic sclerosis (SSc), polymyositis/dermatomyositis, and rheumatoid arthritis, accompanied by the presence of high titers antibodies to U1 ribonucleoprotein (anti-U1RNP). We had reported a case of a male patient who had chronic dysphagia, progressive dyspnea, and the presence of skin lesions. The examination found chronic dysphagia, progressive dyspnea caused by pneumonia and suspected interstitial lung disease, autoimmune hemolytic anemia, discoid lesions, and skin biopsy revealed scleroderma. This patient did not meet the diagnostic criteria of MCTD because anti-U1RNP examination had not been performed as one of the diagnostic criteria' requirements. However, because he has vital signs and symptoms toward MCTD where there were overlapping symptoms of SLE and SSc symptoms, we diagnosed him as MCTD.
\end{abstract}

Keywords: Connective tissue disease, autoimmune, U-RNP antibody.

Correspondence: Awalia

E-mail: awalia_nov74@yahoo.com

Article history: • Received 21 December $2020 \bullet$ Accepted 27 January 2021

\section{INTRODUCTION}

There are six autoimmune connective tissue diseases, which are characterized by the presence of autoantibodies and autoimmune-mediated organ damage, including systemic lupus erythematosus (SLE), systemic sclerosis/scleroderma $(\mathrm{SSc} / \mathrm{Scl})$, polymyositis (PM), dermatomyositis (DM), rheumatoid arthritis (RA), and Sjogren's syndrome (SS) (Bennett, 2017). Mixed connective tissue disease (MCTD) is an overlap disease, has been recognized as an entity disease with a mixture of clinical manifestations from SLE, SSc, $\mathrm{PM} / \mathrm{DM}$, and RA, accompanied by the presence of U1Ribonucleoprotein antibodies (anti-U1-RNP) in hightiters (Ortega-Hernandez \& Shoenfeld, 2012; Tendean et al., 2018). The prevalence of MCTD in the Indian/ Alaska America is 6.4 per 100.000 population (Ferucci et al., 2017). In Japan, 2.7 patients per million population are reported (Tendean et al., 2018). The mean age of onset was 31.9 years, and more than three-quarters of patients were females. This confirms the disease's rarity and predilection for the female sex (Katewa et al., 2014).

Here we present a rare case of a male patient with chronic dysphagia, progressive dyspnea, skin lesions, and autoimmune hemolytic anemia (AIHA). Furthermore, this patient was diagnosed with MCTD.

\section{CASE REPORT}

A man, 52 years old, came to our hospital with chief complaints of shortness of breath and difficulty in swallowing. Since the last two months, the complaint of shortness of breath occurred both during activity and rest, then became worse since last week, and productive cough since last week. There was no complaint of fever. $\mathrm{He}$ also complained of difficulty swallowing for the last eight months and became worse for three months. $\mathrm{He}$ has been given refined food through a nasogastric tube (NGT) to facilitate feeding since last month. He experienced hoarse sounds for six months. There was no complaint of bluish or pale of the fingers when exposed to cold water. He felt the skin on his hands and feet tight since last two months.

It was found in the past medical history since eighteen months ago that the patient experienced a complaint of reddish skin accompanied by scaly and flaking skin on the face and neck area. Besides, there was joint pain in the lower back, waist, wrists, legs, fingers, and toes. Joint pain existed, especially after waking up and feels better during the day after activity. He also complained of frequent hair fall, sometimes mouth ulcers, and redness on the face increases when exposed to sunlight. At that time, he was diagnosed with psoriasis arthritis by a rheumatologist, then received methylprednisolone and sulfasalazine. Because there was no improvement, sulfasalazine was replaced with mycophenolate 
mofetil (MMF). During MMF therapy, the patient's complaints improved. However, he had not taken MMF for the last three months due to limited funds to buy. Then, he experiences deteriorating health conditions. Besides, it was not easy to get suitable blood for transfusion based on the cross-match last month. No family history has the same complaints.

On physical examination, the general condition was weak, GCS E4V5M6. Body mass index 15.62. Blood pressure (BP) $92 / 60 \mathrm{mmHg}$, heart rate (HR) 115 beat/ minute, respiratory rate (RR) 26 times/minute, temperature $36.70 \mathrm{C}$, oxygen $^{-}$saturation (SO2) 95\%. Anemia and dyspnea were found, and less hair because of frequent hair loss. There was no jaundice or cyanosis, no oral ulcer. Salt and pepper appearance was found in the chest. Discoid lesions were found on the ears and the back. Heart sounds normal. The lungs examination was sonor percussion, and the vesicular sound decreased and rhonchi in the lower third of both lungs. There was edema in the inferior extremity.

Laboratory results showed haemoglobin $(\mathrm{Hb}) 8.6 \mathrm{~g} /$ dL, white blood cells (WBC) 8.630/ $\mu \mathrm{L}, 95 \%$ neutrophils, $1.1 \%$ lymphocytes, platelets $124,000 / \mu 1$, erythrocyte sedimentation rate (ESR) 12 , blood glucose $134 \mathrm{mg} / \mathrm{dL}$, albumin $1.84 \mathrm{~g} / \mathrm{L}$, aspartate transaminase (AST) $82 \mathrm{U} / \mathrm{L}$, alanine transaminase (ALT) $57 \mathrm{U} / \mathrm{L}$, blood urea nitrogen 15 $\mathrm{U} / \mathrm{L}$, creatinine $0.47 \mathrm{mg} / \mathrm{L}$, sodium $119 \mathrm{mmol} / \mathrm{L}$, potassium $4.8 \mathrm{mmol} / \mathrm{L}$, chloride $96 \mathrm{mmol} / \mathrm{L}$, non-reactive human immunodeficiency virus, hepatitis $\mathrm{B}$ and $\mathrm{C}$. Blood gas analysis (BGA): $\mathrm{PH} 7.44, \mathrm{PCO} 2$ 35, PO2 86, HCO3 23, BE -0.4, SO2 97\%. Pleural fluid analysis, an impression of exudate. Previous laboratory results, one year ago (when patients had taken immune-suppressants), showed negative antinuclear antibody (ANA) profile. Laboratory results, one month ago (when the patient did not take immune-suppressant), showed $\mathrm{Hb} 6.2$ g/dL, ESR 154/157, high-sensitivity C-reactive protein (hs-CRP) $51.6 \mathrm{mg} / \mathrm{L}$, WBC $8,800 / \mu \mathrm{L}$, platelet $214.000 / \mu \mathrm{L}$, reticulocytes $3.5 \%$, total bilirubin 1.66, direct bilirubin 0.38 , albumin 2.1 g/L, AST $94 \mathrm{U} / \mathrm{L}$, ALT $27 \mathrm{U} / \mathrm{L}$. Blood peripheral smear revealed the blood edge of the autoimmune condition. The cross-matched blood one month ago (when patients did not take immune-suppressants) was positive direct coomb test (DCT). Chest X-ray showed bilateral pleural effusion and pneumonia. The previous chest X-ray, 1 month ago, showed that the lungs revealed reticular pattern in both lungs, suspicion of bilateral interstitial lung disease (ILD) and pleural effusion.

Based on the examination, he was diagnosed with suspected MCTD, chronic dysphagia, suspected ILD, pneumonia, sepsis, hypoalbuminemia, bilateral pleural effusion,AIHA, hypovolemichyponatremia. Hewasplanned to examine acid-fast bacilli, sputum culture, complement (C3 and C4), skin biopsy, esophagogastroscopy, and chest computed tomography (CT). He was given supplemental oxygen, fluid rehydration, intravenous albumin, antibiotic levofloxacin, the leuco-depleted red cell transfusion because of oxygen demand. Methylprednisolone and MMF therapy were postponed until the infection was resolved (it could be started if the CRP $<50 \mathrm{mg} / \mathrm{L}$ ).

\section{Disease Progression}

On the 3rd day of care: He had shortness of breath, weakness, joint pain almost the entire body, BP 100/70 $\mathrm{mmHg}$, HR 105 beat/minute, RR 26 times/minute, SO2 96\% (simple mask). There were anemia and dyspnea. The laboratory results: $\mathrm{Hb} 9.6 \mathrm{~g} / \mathrm{dL}, \mathrm{WBC} 12,600 / \mu \mathrm{L}$, neutrophils $91 \%$, lymphocytes $2.7 \%$, platelets $77,000 /$ $\mu \mathrm{l}$, reticulocytes $1,08 \%$, albumin $2.2 \mathrm{~g} / \mathrm{L}$, sodium 129 $\mathrm{mmol} / \mathrm{L}$, potassium $4.4 \mathrm{mmol} / \mathrm{L}, \mathrm{CRP} 8.6 \mathrm{mg} / \mathrm{dL}, \mathrm{C} 3: 39$ $\mathrm{mg} / \mathrm{L}, \mathrm{C} 4: 24.4 \mathrm{mg} / \mathrm{dL}$, non-reactive HIV, smear sputum: gram-negative bacterias, sputum culture: Pseudomonas aeruginosa. He was given intravenous methylprednisolone $1 \mathrm{mg} /$ body weight, oral MMF 2x360 mg, and antibiotic. He was planned to pulse dose of methylprednisolone and cyclophosphamide if the infection had been resolved.

On the 6th of care: Shortness of breath become worse, BP 100/60 mmHg, HR 110 beat/minute, RR 28 times/ minute, temperature $36.80 \mathrm{C}$, SO2 97\% (non-rebreathing mask). Dyspnea was found, and ronchi in both basal lungs. Laboratory of BGA: PH 7.28, PCO2 77, PO2 107, HCO3 36, BE 9.5, SO2 97\%. The skin biopsy was suggesting scleroderma. He suffered respiratory failure, therefore was planned for a ventilator. However, he refused the ventilator.

On the 7th day of care: Shortness of breath worsened, GCS E3V3M5, BP 80/50 mmHg, HR 120 beat/minute, RR 30 times/minute, SO2 90-93\%. The patient had not been tested for anti-U1-RNP because of limited funds. He eventually died because of septic shock and respiratory failure.

\section{DISCUSSION}

The initial presentation of the MCTD patients usually comprises nonspecific signs such as swollen digits, arthralgia, myalgia or muscle weakness, acid reflux or dysphagia, Raynaud's phenomenon, shortness of breath, general malaise, and fatigue (Katewa et al., 2014). MCTD can be a severe disease with the development of pulmonary arterial hypertension (PAH), glomerulonephritis, vasculitis, gastrointestinal bleeding, and severe central nervous involvement, which does not always have a good prognosis (Ortega-Hernandez \& Shoenfeld, 2012). The basic premise of the MCTD concept and included in the main diagnostic criteria is the presence of anti-U1-RNP antibodies. Other investigations that can be done to assess the involvement of specific organs in the MCTD include routine blood tests, urinalysis, creatine phosphokinase, radiographic of arthritis joints, chest X-ray, electrocardiography, and echocardiography. Echocardiography is the most useful screening test for PAH (Bennett, 2017). Pulmonary functions should be monitored in MCTD because the pulmonary disease is common and the most frequent disease-associated cause of death. High-resolution CT (HRCT) of the chest may be indicated to assess ILD when abnormalities are found on the plain-film radiographs or pulmonary function testing (Hoffman \& Greidinger, 2013). There are four diagnostic criteria for MCTD, such as Sharp criteria, Alarcon-Segovia, Kasukawa, and Kahn\&Appeboom's. A comparative study comparing all four criteria reported that the criteria for Alarcon-Segovia and Kahn had the best sensitivity and specificity (Bennett, 2017).

This patient had clinical signs and symptoms, such as difficulty swallowing, worsening shortness of breath, the skin that feels tight, and AIHA. These were felt after did not take MMF drug since last three months. Past medical history, he has reddish and scaly skin accompanied by joint pain, frequent hair fall, and oral ulcer. At that time, he was diagnosed with psoriatic arthritis and received immunesuppressants, but he did not take immune-suppressants for the last three months. Physical examination revealed anemia, dyspnea, dysphagia, ronchi in the basal lung, salt and pepper appearance, acrosclerosis, and discoid lesions. Laboratory tests revealed anemia, lymphopenia, 
hypoalbuminemia, hyponatremia, and decreased $\mathrm{C} 3$. Serological results of ANA profile revealed negative (during taking immune-suppressant for six months). These negative serological results could also result from false negatives because the patient was examined while taking immune-suppressant. The DCT was positive (when the patient did not take immune-suppressant for three months). Chest X-ray revealed pleural effusion and reticular pattern in both lungs, suggesting diffuse ILD. Sputum cultures revealed Pseudomonas aeruginosa. Skin biopsy revealed scleroderma. The patient was planned for esophagoduodenoscopy, echocardiography, and chest CT, but he did not have the opportunity to do because he died before the examination schedule.

The serology of patients with autoimmune diseases can produce negative results despite showing clear signs and symptoms of the disease. This can be due to the influence of antigenic deficiency in a testing substrate, concurrent immunosuppressive treatment, or persistent profound proteinuria associated with IgG loss through the kidneys. All of these can cause spuriously negative serological test results (Mugunthan, 2005). An elevated anti-U1-RNP is one of the diagnostic criteria of MCTD. The patient did not undergo the test because of limited funds. However, due to the presence of overlapping symptoms between SLE (arthritis with synovitis, discoid lesions, AIHA, decreasing C3, lymphopenia) and Ssc (acrosclerosis, chronic dysphagia, suspicion of ILD, salt and pepper appearance, and skin biopsy revealed scleroderma), we diagnosed this patient as MCTD.

Recently, there were no specific recommended guidelines for the treatment of MCTD. Immunosuppressive and steroid are still the primary therapy for MCTD. The therapy principle is based on clinical manifestations that appear in patients (Tendean et al., 2018). The leading cause of death in patients with MCTD is PAH (Hajas et al., 2013). In a cohort study, it was found that the MCTD phenotype was stable in the majority of patients, but a small proportion of patients who were initially diagnosed with MCTD could develop to other connective tissue diseases. Of the 118 patients, $9 \%$ of MCTD developed to SLE, RA, and SSc in 17 years. Long-term remission in MCTD is not frequent. However, the disease course tends to be milder compared to SLE and SSc (Reiseter et al., 2017). These patients were given supplemental oxygen, antibiotics for pneumonia, corticosteroids, and MMF. He was planned for pulse dose corticosteroid and cyclophosphamide if the infection had been resolved, but this was not given because he died because of septic shock and respiratory failure.

\section{CONCLUSION}

We had reported a case of a 52-year-old man with chronic dysphagia, progressive dyspnea caused by pneumonia and suspected ILD, AIHA, discoid lesions, and skin biopsy revealed scleroderma. This patient did not meet the diagnostic criteria of MCTD because an anti-U1RNP examination had not been performed as one of the requirements. However, because he had vital signs and symptoms toward MCTD, so this patient was diagnosed as MCTD.

\section{CONFLICT OF INTEREST}

The author declare there is no conflict of interest of this study.

\section{REFERENCES}

Bennett, R. M. (2017). "Overlap Syndromes," in Kelley and Firestein's Textbook of Rheumatology, eds. G. S. Firestein, R. C. Budd, S. E. Gabriel, I. B. McInnes, and Ja. R. O’Dell (Philadelphia: Elsevier), 1489-1509.

Ferucci, E., Johnston, J., Gordon, C., Helmick, C., and Lim, S. (2017). Prevalence of mixed connective tissue disease in a population-based registry of American Indian/Alaska Native people in 2007. Arthritis Care Res. 69(8), 68, 1271-1275. doi:10.1002/acr.

Hajas, A., Szodoray, P., Nakken, B., Gaal, J., Zold, E., Laczik, R., et al. (2013). Clinical course, prognosis, and causes of death in mixed connective tissue disease. J. Rheumatol. 40, 1134-1142. doi:10.3899/jrheum.121272.

Hoffman, R. W., and Greidinger, E. L. (2013). Mixed Connective Tissue Disease and Undifferentiated Connective Tissue Disease. 8th ed., eds. D. J. Wallace and B. H. Hahn Philadelphia: Elsevier Available at: http://linkinghub. elsevier.com/retrieve/pii/B9781437718935000364.

Katewa, R., R.S., J., and G.L., B. (2014). Mixed connective tissue disorder: A case report. Int. J. Case Reports Images 5, 650. doi:10.5348/ijcri-2014116-cr-10427.

Mugunthan, M. (2005). Antinuclear antibodies-negative systemic lupus erythematosus - does it exist ? 2005.

Ortega-Hernandez, O. D., and Shoenfeld, Y. (2012). Mixed connective tissue disease: An overview of clinical manifestations, diagnosis and treatment. Best Pract. Res. Clin. Rheumatol. 26, 61-72. doi:10.1016/j.berh.2012.01.009.

Reiseter, S., Gunnarsson, R., Corander, J., Haydon, J., Lund, M. B., Aaløkken, T. M., et al. (2017). Disease evolution in mixed connective tissue disease: Results from a longterm nationwide prospective cohort study. Arthritis Res. Ther. 19, 1-9. doi:10.1186/s13075-017-1494-7.

Tendean, M., Nuriawan, S. A., and Nugroho, P. (2018). Interstitial lung disease in mixed connective tissue disease. Indones. J. Rheumatol. 9, 29-34. doi:10.37275/ijr. v9i1.68. 